\title{
Age and Experience-Dependent Representational Reorganization During Spatial Learning
}

\author{
S. J. Y. MIZUMORI ${ }^{1}$ AND A. KALYANI \\ Psychology Department, University of Utah, Salt Lake City, UT 84112
}

Received 16 April 1996; Revised 10 July 1997; Accepted 2 September 1997

\begin{abstract}
MIZUMORI, S. J. Y. AND A. KALYANI. Age and experience-dependent representational reorganization during spatial learning NEUROBIOL AGING 18(6) 651-659, 1997.-Previously, we found that aged rats showed a significant enhancement of hippocampal CA1 place cell spatial specificity, as well as a reduction of hilar place cell spatial specificity, during asymptote performance of a spatial memory task. Because such an age effect was not observed when animals performed a nonspatial task, the present study tested the hypothesis that the different patterns of spatial selectivity observed in memory and nonmemory tests reflected a redistribution of spatial representations that occurred in response to changing task demands. In the present experiment, after animals became familiar with the test environment and motor demands of performance on a radial maze, CA1 and hilar place cells were recorded as they learned a spatial memory task. CA1 place cells recorded from unimpaired old, but not impaired old or young, animals became more spatially selective as animals learned the task. Hilar spatial selectivity for both age groups was not significantly related to choice accuracy. These data support the hypothesis that at least a subpopulation of aged rats may benefit from reorganization of spatial representations in such a way that the normal age-related spatial learning deficit is attenuated. (c) 1997 Elsevier Science Inc.
\end{abstract}

Place cells Hippocampus Spatial learning Representational neuroplasticity Aging

THE relatively poor performance of aged rats on spatial memory tasks has been shown not to be due to general sensory or motor decline (e.g., 3,6,12,29,33). Rather, arguments have been made for a select difficulty in acquiring new information, especially spatial information. Recent studies have shown that aged rats and monkeys do not show significant loss of cells in any of the hippocampal subregions examined (i.e., the granule cell layer, hilus, CA3, CA1, or subiculum; refs. 28,30,31). Therefore, it is likely that a hippocampal contribution to the spatial learning decline with senescence reflects more subtle changes in hippocampal cell function. Indeed, there is evidence that the synaptic structure of hippocampal neurons undergoes age-associated changes that are correlated with the memory impairment (e.g., ref. 9). The functional significance of such synaptic changes is presently unknown. However, it is not unreasonable to postulate that such intracellular alterations might change the manner in which information is coded by aged hippocampal neurons.

A large proportion of hippocampal neurons (place cells) are known to selectively discharge as animals traverse circumscribed portions of environmental space, referred to as place fields $(24,25)$. Hill and Best (11) showed that place fields emerge relatively quickly when placed in a new environment. This initial result was recently extended by Wilson and McNaughton (32) and Austin and colleagues (1), who showed that place coding by particular hippocampal neurons can undergo initially rapid, then more subtle long-term changes with continued exposure to a constant environment. The latter studies demonstrate that familiarity can influence place cell firing and that place field specificity is not merely a reflection of the current sensory environment.

Previously, we reported that CA1 and hilar neurons recorded from aged rats show different organization of spatial (place) representation compared to young rats when well trained animals perform a spatial memory task (19). Specifically, CA1 cells of aged animals showed enhanced spatial selectivity, and hilar cells demonstrated reduced spatial selectivity. The present study tested the hypothesis that this age-related representational reorganization is experience-dependent. Markus et al. (14) examined the relationship between various place field properties and continued performance on a forced choice (i.e., non memory) radial maze task. A significant relationship between choice accuracy and place cell properties was reported, but this conclusion was based on relatively few "errors" made by the rats. The present study tested more directly how changing the mnemonic requirements of a familiar and spatially complex environment might alter place cell firing not only in young but also aged animals.

\section{METHOD}

\section{Subjects, Surgical Procedures, and Behavioral Training}

Male Fischer-344 rats (retired breeders from Harlan SpragueDawley Laboratory) were obtained at 9 months and 24 months of age. The rats were singly housed and allowed to adapt to laboratory conditions for 2 weeks before surgical implantation of recording electrodes. Mature, young rats were initially anesthetized with $33 \mathrm{mg} / \mathrm{kg}$ Nembutal $(50 \mathrm{mg} / \mathrm{mL})$, and aged rats with 25

\footnotetext{
${ }^{1}$ Address correspondence to: Sheri J. Y. Mizumori, Psychology Department, University of Utah, Salt Lake City, UT 84112.
} 
$\mathrm{mg} / \mathrm{kg}$ Nembutal, followed by supplements of $0.05 \mathrm{~mL}$ as needed. Electrodes were placed at the following stereotaxic coordinates (skull horizontal): (AP) $-3.5-4.5 \mathrm{~mm}$ posterior to Bregma, (L) \pm $2.5 \mathrm{~mm}$ lateral to the midsaggital suture, and (DV) about $1.5 \mathrm{~mm}$ below the dural surface for CA1 recordings, or $2.0 \mathrm{~mm}$ below the dural surface for hilar recordings. Two microdrives were implanted per animal, one over each hemisphere. A reference electrode (Teflon-coated stainless steel) was placed in corpus callosum: (AP) $-6.5 \mathrm{~mm}$; (L) $2.0 \mathrm{~mm}$. A ground lead was soldered to a ground screw attached to the skull. Amphenol pin connectors attached to each electrode were inserted into a connector that became permanently attached to the rat's head. After surgery, 0.1 $\mathrm{mL}$ Bicillin (300,000 units/mL) was administered into each hindleg to prevent infection.

Animals were allowed to recover from surgery for 7 days. After this period, access to food was restricted to maintain animals at about $80 \%$ of their free feeding weights. The rats were then placed on an 8-arm radial maze and trained according to a forced-choice procedure (for details, see ref. 19). Briefly, individual arms of the maze were presented to the rat sequentially and in pseudo random order such that the rat entered each maze arm once per trial. After animals were able to perform 8 such maze trials $(2$ min intertrial interval) within about an hour, and for 7 consecutive days, the recording electrodes were slowly advanced toward or through the hippocampus. When stable, well isolated single hippocampal complex-spike cells were obtained in either the CA1 or hilar regions, the maze training procedure was changed to a spatial memory procedure. That is, for each trial, following the individual presentation of the first 4 maze arms, all 8 arms were simultaneously presented and the rat was required to select those arms not previously entered that trial. The animals were not handled between choices 4 and 5 , and there were no experimenter-imposed delays between choices 4 and 5. Reentries constituted errors. Again, animals performed 8 trials per day for 7 consecutive days. We attempted to record the same cell(s) throughout this spatial learning phase.

We attempted to take run speed into account by calculating the amount of time spent per choice. This was done by taking the total time required to complete the task, divided by the number of choices made by the animal. This was determined for each trial, then averaged across trials to arrive at a score for each day of testing.

\section{Unit Recording and Analysis Procedures}

Hippocampal single unit activity was recorded according to the stereotrode method described by McNaughton and colleagues (17). Specifically, unit activity was independently recorded on two adjacent lacquer-coated tungsten wires $(25 \mu \mathrm{m}$ dia; California FineWire), which were twisted together, dipped in Epoxylite, and then baked. The electrode tips were gold plated to give final impedances of $50-150 \mathrm{k} \Omega$ (tested at $1 \mathrm{kHz}$ ). Each stereotrode pair of wires was threaded through a 30-gauge stainless steel tube, then mounted on a moveable microdrive (19-21). A headstage was attached to a connecting socket on the rat's head for all spatial learning recording sessions. The headstage contained 5 field effect transistors and an infrared LED for behavioral monitoring (20). The incoming signals of the stereotrode pair were processed independently, with an amplification of 5-10 K, and filtered at $600-800 \mathrm{~Hz}$ (high pass) and $6 \mathrm{kHz}$ (low pass). The analog signals were subsequently passed through a window discriminator such that a 1-ms sampling period by a DataWave system began when a signal from either channel surpassed a predetermined threshold. The maximum and minimum voltages of each signal, as well as the latencies of these values from the onset of the sampling period, were used to preliminarily isolate single spike generators within an otherwise multiunit record. More detailed off-line analyses were available to further isolate single units (e.g., template matching algorithms; ref. 19).

The final recording depths were determined relative to stratum pyramidale, which could be clearly identified by its electrophysiological properties. CA1 neurons were recorded in the first layer of complex-spike cells encountered, which was typically about 2000 $\mu \mathrm{m}$ below the cortical surface. Hilar complex-spike cells were typically encountered 700-1000 $\mu \mathrm{m}$ below CA1. For CA1-recorded animals, the electrodes entered CA1 only. For hilarrecorded animals, the electrodes passed through CA1 first, then the dentate region. The hilar group of cells likely included a combination of granule cells, hilar cells, and possibly CA4 cells. Whether a particular animal contributed CA1 or hilar data was determined somewhat randomly at the time of surgery. As a result, the order of recording was mixed. The stereotrodes were lowered through one hemisphere at a time; if well isolated units were not encountered in the first hemisphere, the stereotrodes were lowered through the second hemisphere.

Spatially localized discharge by hippocampal CA1 and hilar neurons was quantified as in previous studies (19-21). Briefly, the firing rates as the rat moved inward and outward on the 8 maze arms were calculated. The highest of these 16 rates was divided by the average of the remaining 15 to arrive at a spatial specificity score. It is important to note that our calculations incorporate not only the location component of spatial behavior but also directional movement. Both aspects of navigational behaviors are particularly characteristic of hippocampal place cells when tested on a radial maze. A cell was considered to have a place field if the cell had a specificity index greater than 2.0. Reliability of a place field was determined by counting the number of trials in which the in-field firing rate was higher than the firing rate on the remaining 7 maze arms. A directional score was calculated for each cell for each day it was recorded; the mean rate was calculated for times when the rat moved inward and outward on the arm associated with the highest rate (i.e., contained the place field). The highest of these two rates was divided by the lowest rate.

\section{RESULTS}

The average number of errors made per trial on a given day of training was compared to a number of single unit firing properties of complex-spike units recorded on the same day. Initial, on-line classification of a complex-spike cell as either a CA1 or hilar neuron was confirmed by examination of the location and depth of the relevant electrode tract in histologic sections. All electrodes passed through dorsal hippocampus.

Ten young and 10 old rats were trained on the forced choice task for $6.5 \pm 1.5$ days and $11.2 \pm 1.6$ days, respectively, before the 7 days of asymptote performance was observed. This difference in the number of days to required to perform the required 8 daily trials was marginally significant, $\mathrm{F}(1,18)=4.38, p=0.05$. Both young and old rats performed at asymptote on the forced choice task for at least 7 days prior to spatial memory training, if not longer. Such training began only when well isolated CA1 or hilar complex-spike cells were encountered. The total number of days of exposure to the test room before unit recording began was found not to vary significantly between the 4 groups: young CA1-21.8 \pm 3.4 days; young hilus-18.2 + 1.5 days; old CA1$26.3 \pm 2.3$ days; old hilus-19.3 \pm 1.2 days. No significant differences existed between age groups or recording sites.

Figure 1 shows that across the 7-day acquisition phase of spatial memory training, both age groups of rats showed significant learning, $\mathrm{F}(6,18)=52.18, p<0.001$ (repeated measures 


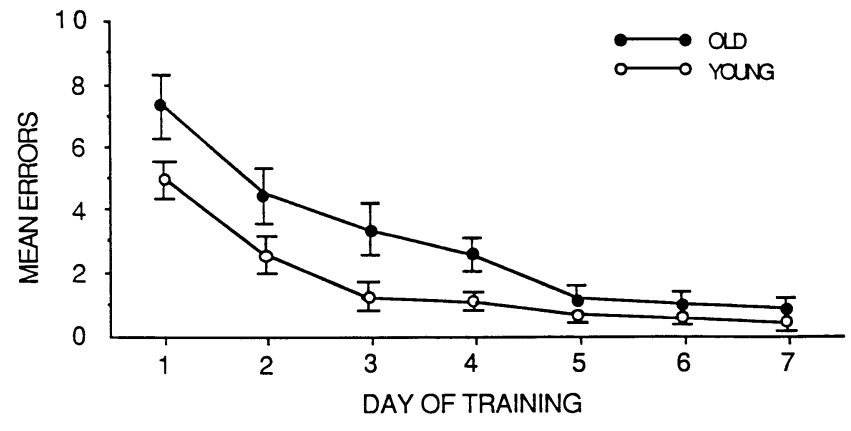

FIG. 1. Mean $( \pm \mathrm{SE})$ number of errors made per trial across the seven days of acquisition of the spatial memory task. Aged rats made significantly more errors overall, and both young and aged rats showed significant learning during this period. In addition, a significant interaction effect of age and days of training suggest that aged rats learned the task at a slower rate than young rats.

ANOVA). In addition, a significant age effect was observed, indicating that overall, the old rats performed worse than young rats, $\mathrm{F}(1,18)=6.38, p<0.05$. Furthermore, a significant interaction effect, $\mathrm{F}(6,18)=2.79, p<0.02$, revealed that aged rats learned the maze task at a slower rate than young rats. This pattern of statistical results replicates that reported previously (19) for young and old rats trained under identical conditions.

A particular unit was considered to have been recorded across days if it was determined that its cluster parameter boundaries were comparable across days. Thus, $19 \%(5 / 26)$ of CA1 complexspike cells and $25 \%$ (10/40) of hilar complex-spike cells recorded from young rats were deemed stable across the 7-days of acquisition training. About 56\% (24/43) of CA1 complex-spike cells and $27 \%(4 / 15)$ of hilar complex-spike cells recorded from old rats were stable across all 7 test days. Other neurons were recorded for 2-6 days. The analysis described below was carried out separately for cells recorded across all 7 days and those that were recorded for less than 7 days. The latter group consisted of cells that were initially recorded on Day 1, then were lost before the 7 days of training were completed, as well as cells that emerged for the first time after Day 1. Unit-behavioral correlates or spike discharge patterns did not significantly differ depending on the duration of recording of a particular unit, or the day of first recording. Therefore, unless otherwise specified, individual recording sessions for each cell were treated as independent events for statistical purposes.

The log values of the spatial selectivity scores of CA1 and hilar complex-spike cells were correlated with the number of errors made by young and old rats. Specifically, the mean specificity for all complex-spike cells recorded on a given day was correlated with the corresponding mean number of errors committed per trial on the same day. The results for CA1 neurons are shown in Figure 2. CA1 complex-spike cells of young rats $(n=4)$ did not show spatially selective firing that was statistically correlated with the number of errors made $(r=+0.17$, df $=26, p>0.10)$. However, reliability was marginally correlated with the number of errors made $(r=+0.39$, df $=26, p=0.05)$. That specificity was not directly related to learning is consistent with our previous result
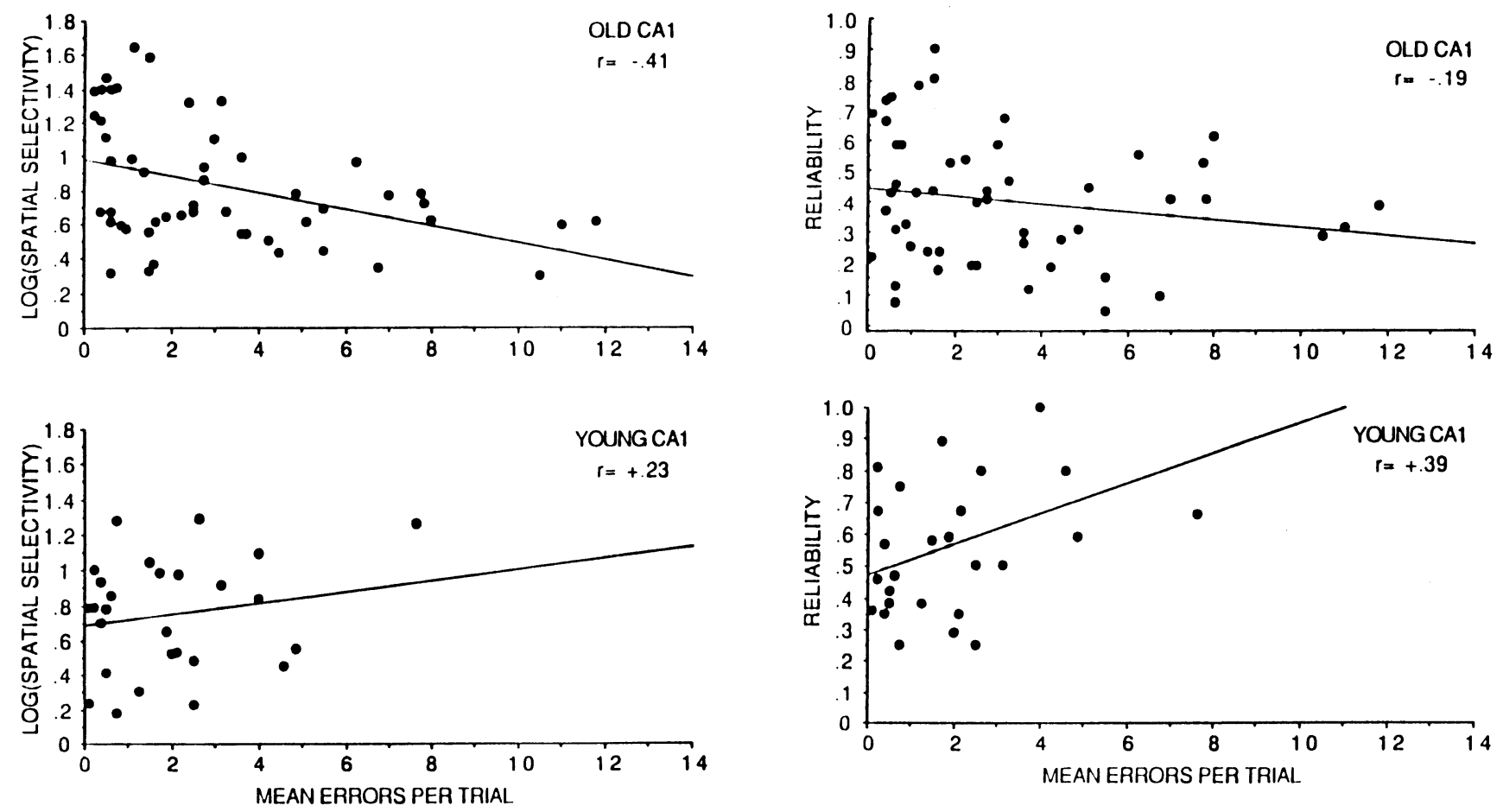

FIG. 2. (LEFT) Scatter distribution showing the correlation between the log transform of spatial selectivity scores of CA1 hippocampal place neurons and the mean number of errors made per trial per day that each cell was recorded. The data presented reflects a summary of data across the entire acquisition period. Each dot represents the average specificity for all cells recorded within a given recording session. A significant correlation was observed between decreasing errors and increasing spatial selectivity for old (TOP), but not young (BOTTOM) rats. (RIGHT) Scatter distribution of the reliability scores of the same cells presented to the left. No relationship was found between reliability scores and errors in old rats; however, a marginally significant positive correlation was observed for young rats. 

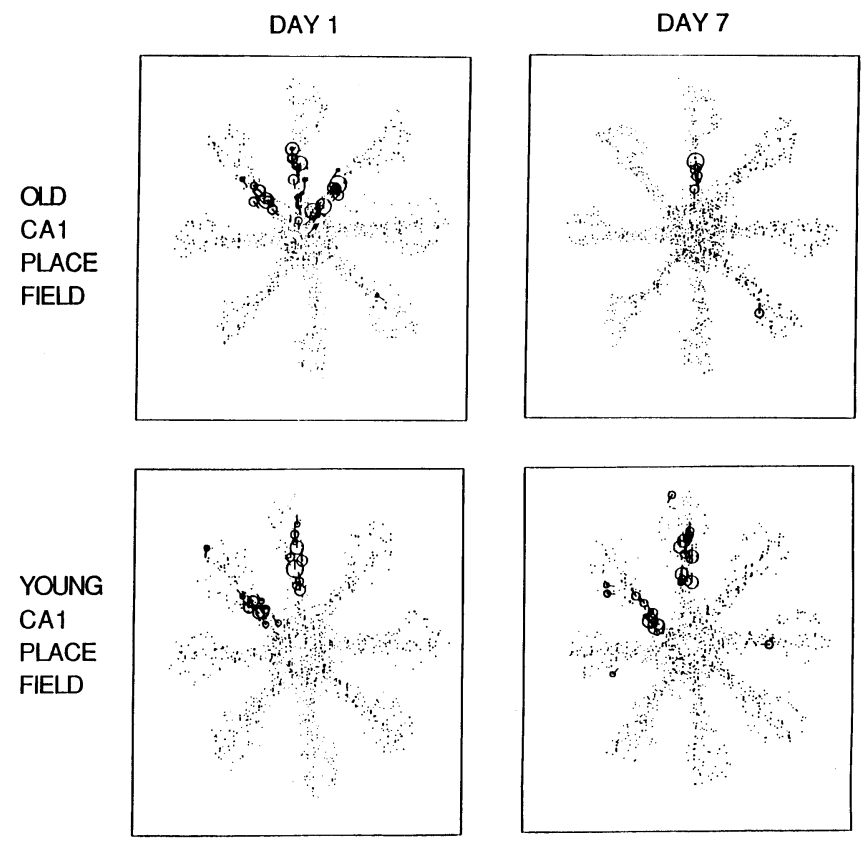

FIG. 3. Examples of the spatial distribution of CA1 place fields recorded from a representative neuron of an aged $(T O P)$ and a representative neuron of a young (BOTTOM) animal on Day 1 (LEFT) and Day 7 (RIGHT) of acquisition of the spatial memory task. Dots indicated visited locations by the rat on the maze. The size of the circles is proportional to the local firing rate of the cell, and the vector indicated the direction of movement by the animal as the cell fired.

(19) that spatial selectivity of young CA1 cells is not different for rats performing at asymptote on a nonspatial task vs. a spatial memory task. It is also consistent with the report by Markus et al. (14) that reliability, and not spatial selectivity, of CA1 place field firing is related to errors. However, it should be noted that the latter authors reported a negative relationship, whereas we found a marginally significant positive relationship, although visual inspection of the reliability data in Figure 2 does not reveal a particularly striking positive correlation.

In contrast to the results from young animals, place-specific firing of CA1 complex-spike cells recorded from old rats $(n=7)$ was significantly correlated with the number of errors made on the maze $(r=-0.41$, df $=50, p<0.01)$. That is, for old, but not young rats, CA1 spatially-selective discharge become more location and/or directionally tuned as learning took place (as indicated by the reduction in errors). Similar to our past results, the mean spatial selectivity score was greater for old rats (young: $7.47 \pm$ 1.9; old: $11.83 \pm 2.6 ; \mathrm{p}<0.05$ ) by the end of acquisition training on the memory task (See Fig. 3 for an example). This age-related increase in spatial selectivity may be due to a constriction of place fields over time because 1) the proportion of aged CA1 cells showing place fields did not change (Day $1=79 \%$ and Day $7=$ $81 \%$ ), and 2) the same relationship between increased spatial selectivity and reduced errors was observed when the analysis was restricted to those cells recorded across the entire 7-day acquisition period $(r=-0.23$, df $=158, p<0.01)$. Furthermore, the average percent change in CA1 place field specificity from Day 1 to Day 7 was $-19.0 \% \pm 18.0 \%$ for young animals and $+91.4 \% \pm 39 \%$ for old animals. There were no effects of age on the reliability of CA1 place fields $(p>0.10)$, and no correlation between errors and place field reliability $(r=-0.19$, df $=50, p>0.10)$.
Many studies have shown that certain physiological functions change only for old rats that show a spatial memory impairment, but not for aged rats whose performance is comparable to young animals (e.g., see ref. 7 for review). Therefore, it was of interest to divide the aged animals into impaired and unimpaired groups to determine if the apparent reorganization of representations in CA1 is related to efficiency of performance. Because young animals made between 0.08 and 0.38 errors per trial by the end of acquisition training, aged animals who averaged more than 0.38 errors per trial at the end of the acquisition period were considered impaired. Figure 4 presents separate analyses for the 4 impaired and 3 unimpaired aged rats who contributed CA1 data. A significant correlation between choice accuracy and spatial selectivity was observed only for the intact, unimpaired aged rats $(r=-0.67$, $\mathrm{df}=20, p<0.01)$. Impaired aged rats did not show such a correlation $(r=-0.22$; df $=29, p>0.10)$. Unimpaired aged rats showed a marginal relationship between errors made and reliability $(r=-0.43$, df $=20, p=0.05)$, although impaired old rats did not $(r=+0.01$, df $=29, p>0.10)$.

Spatial selectivity scores represent a comparison between in-field and out-of-field firing rates. Because we found a significant age effect on spatial selectivity scores for CA1 cells, it was of interest to determine whether this was due to changes in the in-field and/or out-of-field firing rates. No significant correlations were found for either young or old CA1 cells with respect to in-field or out-of-field firing rates. This raised the possibility that different patterns of firing rate changes occurred in the aged CA1 cells. Some cells may have increased in-field firing coincident with decreased out-of-field firing; others may have increased in-field firing only, or decreased out-of-field firing only. In all cases, the result would be increased spatial selectivity. An initial examination of these possibilities was performed by evaluating further those 24 aged CA1 place cells that were held for the duration of the acquisition period. Specifically, the proportion change in in-field firing rate and out-of-field firing rate from Day 1 and Day 7 was first calculated according to the following formula: $(a-b) /(a+$ $b$ ), where $a=$ Day 1 rates and $b=$ Day 7 rates. Figure 5 presents a scatterplot of the resultant indices of proportion change: positive values indicate increased firing by Day 7 , and negative values indicate decreased firing by Day 7 , for either in-field (x axis) or out-of-field (y axis) rates. These data show that although different patterns of change occurred in our old animals, the change was observed for both in- and out-of-field rates. Further, there was a general tendency for the in-field firing rate to change more than the out-of-field firing rate. This pattern supports the hypothesis that place fields of aged CA1 cells tended to become smaller.

Figure 6 shows that in contrast to what was observed for cells recorded in the CA1 region, place-specific firing by hilar complexspike cells was not correlated with the number of errors made on the maze for either young $(n=6)$ or old $(n=3)$ rats (young: $r=$ +0.18 , df $=41, p>0.10$; old: $r=-0.05$, df $=16, p>0.10$ ), regardless of their memory status. Nevertheless, the mean spatial selectivity scores at the end of training showed the same age and experience-dependent pattern reported previously: $11.07 \pm 4.6$ and $5.08 \pm 0.7(p<0.05)$ for young and old rats, respectively.

A correlation has been suggested to exist between spike amplitude and spatial selectivity, with larger spikes being associated with greater selectivity (32). It has also been suggested that spatial selectivity scores could be biased by the overall spontaneous firing rate of neurons (4). These suggestions, together with the concern that the spike signal might have deteriorated over multiple recording sessions, prompted us to determine if the observed age-related change in CA1 place field spatial selectivity could be accounted for by age-dependent changes in spike amplitude and/or mean firing rate. Considering those cells that were recorded across 

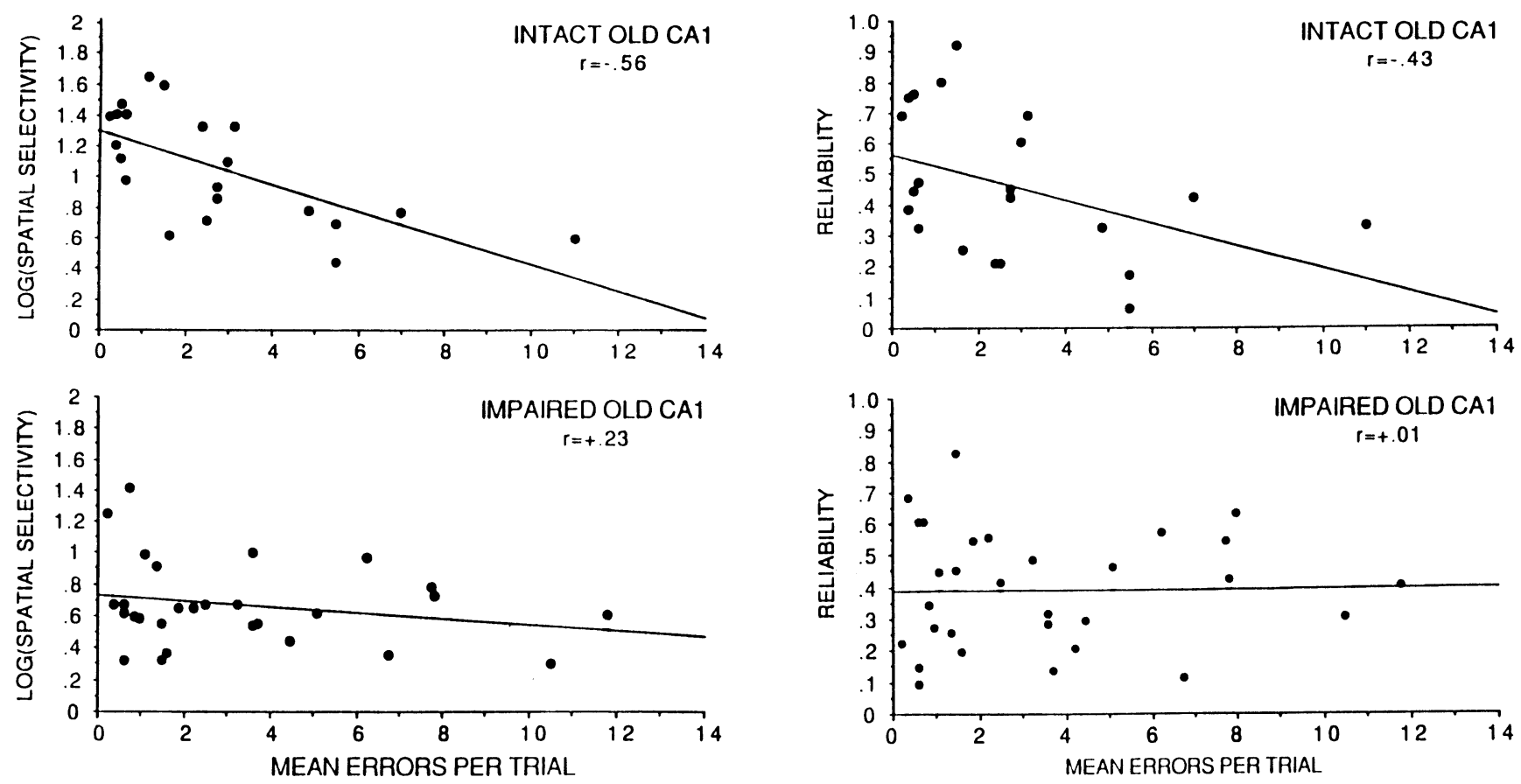

FIG. 4. Scatter distribution showing the correlation between the log transform of spatial selectivity (LEFT) and reliability (RIGHT) scores of CA1 hippocampal place neurons and the mean number of errors made per trial for memory-intact old rats $(T O P)$ and memory-impaired old rats $(B O T T O M)$. Only the memory intact old rats showed a significant correlation between choice accuracy and spatial selectivity scores, and between choice accuracy and spatial reliability. One interpretation of this pattern of results is that such representational reorganization facilitated accurate performance on the memory task.

all 7 acquisition training days, it was found that the average CA1 complex-spike cell amplitudes for Days 1 and 7 for young animals were $168.8 \mu \mathrm{V} \pm 21.5 \mu \mathrm{V}$ and $165.8 \mu \mathrm{V} \pm 26.0 \mu \mathrm{V}$, respectively. CA1 complex-spike cell amplitudes for cells recorded from old

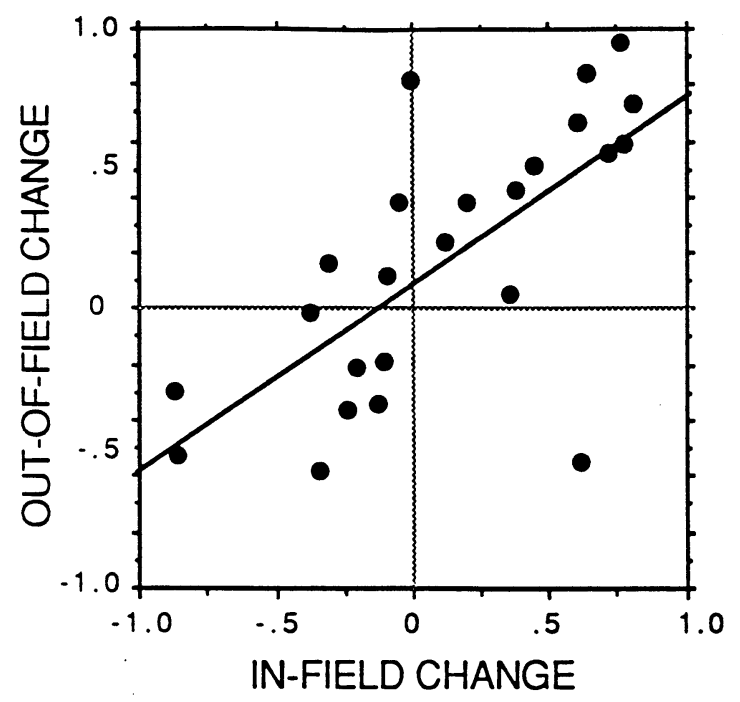

FIG. 5. A comparison of the proportion change in in-field firing rates and out-of-field firing rates from Day 1 to Day 7 of acquisition training for those aged CA1 place cells that were held across the training period. It is clear that different patterns of change were observed and ultimately contributed to the correlations shown in Figure 2. animals on Days 1 and 7 were $170.7 \mu \mathrm{V} \pm 18.7 \mu \mathrm{V}$ and 139.7 $\mu \mathrm{V} \pm 15.1 \mu \mathrm{V}$, respectively. For both age groups, the change in amplitude over days was not statistically significant. Also, no age effects on spike amplitude were observed when all CA1 cells were considered together, regardless of the number of days a cell was recorded. A similar pattern of results was also obtained for hilar cells. Thus, a change in spike amplitude across days could not readily account for the age-related elevation of place specificity of CA1 complex-spike cells, nor the reduced specificity of hilar complex-spike cells. However, this pattern of results is entirely consistent with our finding that unit spatial selectivity and reliability of newly encountered cells did not differ from correlates of cells that were recorded over many days (see above).

The above described spike amplitude data are congruent with the claim by McNaughton and colleagues (32) that a significant correlation exists between spike amplitude and spatial selectivity score. Such a correlation was observed for both CA1 and hilar cells of young rats when one correlated a given day's specificity score with the average spike amplitude for that same cell on the same day $(r=+0.24$, df $=99, p<0.02$ and $r=+0.26$, df $=142, p$ $<0.01)$. A similar correlation was also observed for hilar cells of old rats $(r=+0.37$, df $=59, p<0.01)$, but not for CA1 cells recorded from aged rats $(r=-0.04$, df $=230, p>0.50)$. It is worth noting here that although spike amplitude changes per se cannot account for the correlation observed for the old CA1 place cells, it is not clear what one can say regarding the significant correlations found. Spike amplitude can vary for a number of reasons, such as distance from the electrode tip, health of the cell being recorded, and electrode impedance.

Mean firing rates did not distinguish the two age groups for either CA1 complex-spike cells (young $=0.53 \pm 0.05 \mathrm{~Hz}$; old $=$ $0.63 \pm 0.13 \mathrm{~Hz}$ ) or hilar complex-spike cells (young $=0.30 \pm$ 

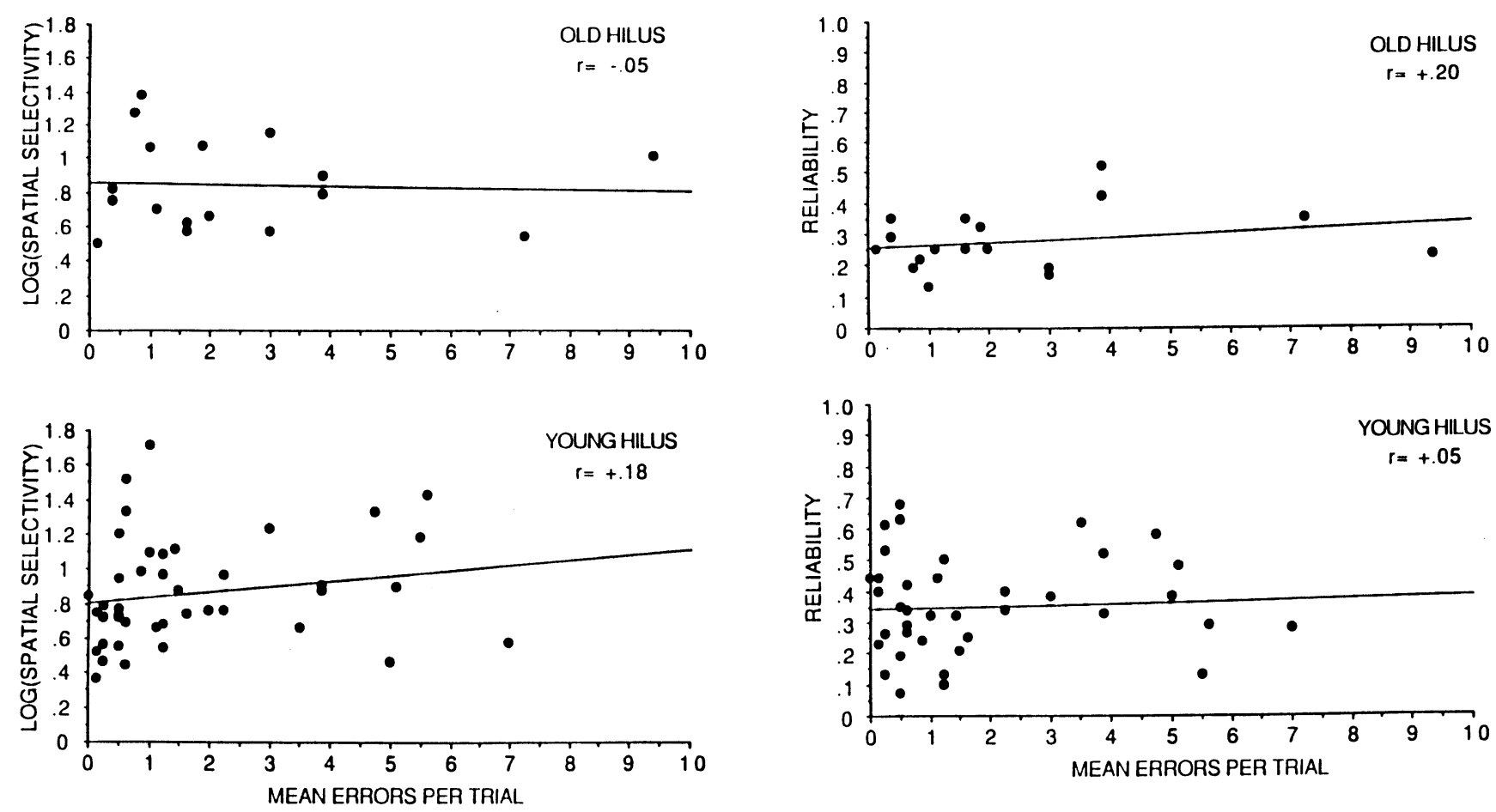

FIG. 6. Scatter distribution showing the correlation between the log transform of spatial selectivity scores of hilar place neurons and the mean number of errors made per trial per day that each cell was recorded. The data presented reflects a summary of data across the entire acquisition period. Each dot represents the average specificity for all cells recorded within a given recording session. No significant correlations were observed between errors and spatial selectivity $(L E F T)$ or reliability $(R I G H T)$ for old (TOP) or young (BOTTOM) rats.

$0.05 \mathrm{~Hz}$; old $=0.23 \pm 0.07 \mathrm{~Hz}$ ). However, mean rates were statistically correlated with spatial selectivity for both CA1 $(r=$ -0.34 , df $=99, p<0.01)$ and hilar cells $(r=-0.18, \mathrm{df}=142$, $p<0.05$ ) of young rats, but not for either cell type recorded from aged rats $(r=-0.12$, df $=230, p<0.08$ and $r=-0.19$, df $=$ $59, p>0.10)$. These findings support previous claims of a relationship between spatial selectivity and mean firing rate in young animals (4), and reveal that the age-specific response of CA1 and hilar neurons to acquisition of a spatial task cannot be accounted for by age-changes in mean firing rate per se.

One consistent feature of hippocampal place cells recorded as animals perform the radial maze has been the directional nature of the spatial code $(13,16)$. Because it has been reported that place fields do not have a significant directional component when rats traverse open arenas (22), it is possible that place fields become more directional when directed search is required (13). If this were true, one might expect a correlation between directional bias of place fields and choice accuracy during acquisition of a spatial memory task. There was no correlation between the latter ratio and errors made $(p>0.10)$ for young or aged animals, nor was there a consistent change in directionality across the days of acquisition training for either young or old CA1 or hilar cells $(p>0.10)$. Comparison of directional indices revealed that CA1 and hilar complex-spike cells recorded from both young and old animals showed clear directional discharge, even on Day 1 of spatial memory training (young CA1: $7.78 \pm 1.76$; old CA1: $10.06 \pm$ 3.04; young hilus: $6.10 \pm 2.20$; old hilus: $6.09 \pm 2.12$ ).

Many reports in the literature show that aged rats typically require more time to perform tasks that require unrestrained navigation. The requirement for more time by old rats could be due to the fact that old rats move more slowly, spending less time in theta-related behaviors. To provide a preliminary evaluation of the relationship between run speed and spatial selectivity, we examined the correlation between spatial selectivity scores with the time required per choice. No statistically significant effects were observed for CA1 or hilar cells of young or old rats (for all, $p>$ 0.10). Furthermore, run time per choice did not significantly vary across the 7 days of acquisition for either young or old animals (repeated measures ANOVA: for both, $p>0.10$ ).

\section{DISCUSSION}

To address the issue of whether the apparent age-related reorganization of spatial representation in hippocampus (19) is due to the fact that, relative to young animals, old rat hippocampal neurons are differentially responsive to task demands, we attempted to correlate the number of errors made during the acquisition of a spatial task and measures of spatial encoding by hippocampal place cells. It was found that CA1 cells recorded from memory-intact old rats became more spatially selective as fewer errors were made on the maze. In contrast, CA1 cells of young animals or of memory-impaired old animals did not show such a correlation. Additionally, hilar place neurons from young or old rats did not show significant correlations between place field specificity and errors. This pattern of effects is consistent with our previous findings of no differences between young and old CA1 or hilar place cells when rats perform a nonspatial task, and significantly increased spatial selectivity of aged CA1 place fields during 
asymptote performance on a spatial memory task. The present data suggest that the age difference in CA1 place representation emerges as new (spatial memory) task demands are placed on the animal.

During acquisition of the new spatial memory task, CA1 place field specificity in old rats was related to errors. This was not the case for aged rats performing at asymptote (19). That acquisition and asymptote performance are differentially related to place field specificity in old rats suggests a different role for CA1 place field in spatial performance depending on whether animals are learning new spatial information or are using flexibly familiar spatial information.

In contrast to what was observed for CA1 place cells, hilar place cell specificity or reliability were not related to choice accuracy. One could argue that the old hilar cells did not show such a correlation because there was a floor effect on spatial selectivity scores. This is probably unlikely because our previous work has shown that spatial selectivity scores of hippocampal neurons as low as 1.0 can be found. A different interpretation is that with age, there are neuropathological changes in the dentate gyrus that must be compensated for (perhaps in CA1) during new spatial learning.

The pattern of effects observed in this study underscores the selective nature of the aging process on hippocampal function $(2,19)$ : there is not a general demise in information processing capacity by all hippocampal neurons. Rather, it appears that subpopulations of aged hippocampal cells have the capacity to increase or decrease the specificity of information codes depending on the behavioral context. This type of experience-dependent plasticity differs from that observed in young animals whose behavioral accuracy is reflected more in terms of changes in reliability, not specificity, of the spatial code. Nevertheless, for both young and old animals, the flexibility conferred by the dynamic nature of spatial codes allows experience and task demands to direct the organization of neural representations such that particular spatial strategies can be implemented.

The possible spatial strategies an animal might use include ones that rely predominantly on global geometric environmental cues, specific environmental landmarks, or idiothetic (internally-generated) cues. In principle, use of any of these strategies could lead to significant learning. There is much experimental data supporting theories that suggest young adult rats use a combination of these strategies to achieve a consistently high level of performance across time. The hippocampus likely contributes to the functional operation of each one of the above spatial strategies because place fields have been shown to respond to changes in global environmental cues, specific landmark cues, and vestibular input $(10,15$, 26,32 ). Not only do hippocampal place cells respond to changes in sensory input, but it is becoming more clear that hippocampal spatial representations of young rats also dynamically reflect the current behavioral context (e.g., 1,19,32). We extend this view of hippocampal representation by hypothesizing that behavioral context determines the relative contribution of different spatial cognitive strategies to behavior of young animals, and that the particular strategy selected reflects the organization of spatial neural codes (19), i.e., the current configuration of neural representation across hippocampal subregions, and the current constellation of encoding properties. Thus, a given cell's correlate may become more or less reliable depending on the relative weight of the message to be relayed. As young adult animals learn a spatial task, certain patterns of hippocampal discharge become reinforced at some level in the spatial learning system (not necessarily in hippocampus), leading to the observed correlation between errors and reliability of spatial codes.
Old animals suffer from a variety of neuropathological changes across different brain structures, including hippocampus. Such changes may lead to a breakdown in the system that modulates the reliability of hippocampal spatial codes, which in turn leads to the development of compensatory information processing networks. The functional consequence of the development of such networks could be a greater reliance on other spatial coding parameters besides reliability, such as the spatial selectivity of cells. That such a change in the nature of hippocampal spatial representation could result in efficient performance is suggested by the results of Figure 2 , which demonstrates that only memory-intact aged rats showed the statistical correlation between errors and place field specificity. Impaired aged rats did not. Given that the hippocampus of old rats probably does not undergo a significant loss of neurons $(28,30)$, enhanced spatial selectivity by CA1 neurons more than likely reflects increased dendritic extent (27), a change in afferent input and/or biophysical/integrative properties of individual CA1 neurons.

Not only did the old rats of this study show increased specificity of CA1 place fields during maze acquisition, but we again observed the result that hilar place fields are less spatially selective. Therefore, we would hesitate to conclude that only CA1 shows a neuroplastic response to learning in old rats. Rather, we suggest there is a reorganization of spatial representation among subpopulations of hippocampal place neurons. Given our presently limited understanding of the significance of place field size, one can only speculate on the significance of this apparent representational reorganization. It was suggested that the clearly different place field sizes of young CA1 and hilar neurons reflect the differential contribution of these substructures to hippocampal processing. Specifically, it may be that CA1 tends to show more broad place fields than hilar place cells because CA1 encodes more than sensory-defined spatial information. CA1 may also encode nonspatial (e.g., contextual) information. In this way, CA1 could provide the hippocampus with a general spatial referent, or framework, within which hilus can encode specific locations. With regard to the lifespan changes observed in aged animals, it is possible that the originally very selective hilus place fields lose spatial specificity because of the deafferentation of the dentate gyrus, which is known to occur (8). Aged CA1 neurons then become more location-selective (perhaps with the support of direct entorhinal afferents (34)), as if to compensate for the lost hilar spatial codes. A functional implication of this series of events is that the aged hippocampus as a whole engages in less efficient and/or less flexible spatial context coding. One prediction of this interpretation is that aged rats should have more difficulty distinguishing changes in behavioral context. Consistent with this prediction is the finding that old rats have more difficulty learning a spatial task in a familiar room where a nonspatial task was previously performed than learning the same spatial task in a novel environment (19).

In the face of past reports of reduced neuroplasticity in the hippocampus of old rats (e.g., ref. 5), it may initially appear contradictory that here we argue that hippocampal neurons of at least some old rats engage in adaptive neural responses. Past work suggesting reduced plasticity generally evaluate dentate gyrus field potential responses to afferent stimulation. Our data are entirely consistent with these previous reports because we found hilar neurons to show lower place field specificity that is not related to errors. Therefore, to elaborate a point raised earlier, the sum of the neurophysiological data with performing old animals suggests that old age not only results in selective deterioration within the hippocampus, but also selective enhancement of cells' relationships to behavior. 
With respect to experiments performed on young animals only, it is worth noting that recently it was reported that hippocampal place cells are more directional when directed search is required (13). Here, we found that directional tuning did not change as animals learned a spatial task subsequent to learning a nonspatial task on the same apparatus. Together with the findings of Markus et al., these data suggest that the directional component of hippocampal neuron discharge is established upon initial exposure to a new environment and/or when new routes to reward must be learned. If the sensory environment or the routes to places of significance (e.g., chocolate milk reward) are unchanged, the directional code is constant. Because the memory demands of a behavioral situation play little or no role in determining the extent of directional bias of these cells, we suggest that the directional component of place field firing reflects knowledge of the general spatial framework, whereas the reliability or selectivity component reflects context-specific landmark information. The implication here is that individual hippocampal place neurons serve as perhaps one of many links between spatial reference frames, leading to integration of specific landmark cues with general environmental information.

A final implication of this work is that it argues for a dynamic and more expansive neural systems approach to the study of the neurobiology of age-related behavioral change. That is, it should not be assumed that the declining function of one population of cells indicates that an entire structure is similarly deteriorating. Rather, given the powerfully dynamic responses of cortical neurons to experience or neural injury $(18,23)$, one should consider the possibility that there may be functional restructuring of information codes that allow animals to behaviorally compensate for age-related neuropathology.

\section{ACKNOWLEDGEMENTS}

This research was supported by National Institutes of Health Grant AG09299 to S.J.Y.M. We gratefully acknowledge the assistance of Karen Burk, Mitch Burt, Sonal Patel, and Leigh Hardy during behavioral testing and data analysis.

\section{REFERENCES}

1. Austin, K. B.; White, L. H.; Shapiro, M. L. Short- and long-term effects of experience on hippocampal place fields. Soc. Neurosci. Abstr. 19:797; 1993.

2. Barnes, C. A. Normal aging: regionally specific changes in hippocampal synaptic transmission. Trends Neurosci. 17:13-18; 1994.

3. Barnes, C. A.; Green, E. J.; Baldwin, J.; Johnson, W. E. Behavioral and neurophysiological examples of functional sparing in senescent rat. Can. J. Psychol. 41:131-140; 1987.

4. Barnes, C. A.; McNaughton, B. L.; Mizumori, S. J. Y.; Leonard, B. W.; Lin., L.-H. Comparison of spatial and temporal characteristics of neuronal activity in sequential stages of hippocampal processing. Prog. Brain Res. 83:287-300; 1990.

5. deToledo-Morrell, L.; Geinisman, Y.; Morrell, F. Individual differences in hippocampal synaptic plasticity as a function of aging: Behavioral, electrophysiological and morphological evidence. In: Petit, T. L.; Ivy, G. O., eds. Neural Plasticity: A Lifespan Approach. New York: Alan R. Liss; 1988:283-328.

6. Gage, F. H.; Dunnett, S. B.; Bjorklund, A. Age-related impairments in spatial memory are independent of those in sensorimotor skills. Neurobiol. Aging 10:347-352; 1989.

7. Gallagher, M.; Nagahara, A. H.; Burwell, R. D. Cognition and hippocampal systems in aging: Animal models. In: McGaugh, J. L.; Weinberger, N. M.; Lynch, G., eds. Brain and Memory: Modulation and mediation of neuroplasticity. New Jersey: Erlbaum; 1995:103126.

8. Geinisman, Y.; Bondareff, W.; Dodge, J. T. Partial deafferentation of neurons in the dentate gyrus of the senescent rat. Brain Res. 134:541$545 ; 1977$.

9. Geinisman, Y.; de Toledo-Morrell, L.; Morrell, F. Aged rats need a preserved complement of perforated axospinous synapses per hippocampal neuron to maintain good spatial memory. Brain Res. 398:266-275; 1988.

10. Gothard, K. M.; Skaggs, W. E., Moore, K. M., McNaughton, B. L. Binding of hippocampal CA1 neural activity to multiple reference frames in a landmark-based navigation task. J. Neurosci. 16:823-835; 1996.

11. Hill A. J.; Best, P. J. Effects of deafness and blindness on the spatial correlates of hippocampal unit activity in the rat. Exp. Neurol. 74:204-217; 1981.

12. Lowry, A. M.; Ingram, D. K.; Olton, D. S.; Waller, S. B.; Reynolds, M. A.; London, E. D. Discrimination learning requiring different memory components in rats: Age and neurochemical comparisons. Behav. Neurosci. 99:638-651; 1985.

13. Markus, E. J.; Qin, Y.-L.; Leonard, B.; Skaggs, W. E.; McNaughton, B. L.; Barnes, C. A. Interactions between location and task affect the spatial and directional firing of hippocampal neurons. J. Neurosci. 15:7079-7094; 1995

14. Markus, E. J.; Barnes, C. A.; McNaughton, B. L.; Gladden V.; Skaggs, W. E. Spatial information content of hippocampal CA1 neurons: Effects of visual input. Hippocampus 4:410-421; 1994.

15. Matthews, B. L., Campbell, K. A.; Deadwyler, S. A. Rotational stimulation disrupts spatial learning in fornix-lesioned rats. Behav. Neurosci. 102:35-42; 1988.

16. McNaughton, B. L.; Barnes, C. A.; O'Keefe, J. The contribution of position, direction, and velocity to single unit activity in the hippocampus of freely-moving rats. Exp. Brain Res. 52:41-49; 1983.

17. McNaughton, B. L.; O'Keefe, J.; Barnes, C. A. The stereotrode: A new technique for simultaneous isolation of several single units in the central nervous system from multiple unit records. J. Neurosci. Meth. 8:391-397; 1983.

18. Merzenich, M. M.; Kaas, J. H.; Wall, J.; Nelson, R. J.; Sur, M.; Felleman, D. Topographic reorganization of somatosensory cortical areas $3 \mathrm{~b}$ and 1 in adult monkeys following restricted deafferentation. Neurosci. 8:33-55; 1983.

19. Mizumori, S. J. Y.; Lavoie, A. M.; Kalyani, A. Redistribution of spatial representation in the hippocampus of aged rats performing a spatial memory task. Behav. Neurosci. 110:1006-1016; 1996.

20. Mizumori, S. J. Y.; McNaughton, B. L.; Barnes, C. A.; Fox, K. B. Preserved spatial coding in hippocampal CA1 pyramidal cells during reversible suppression of CA3 output: Evidence for pattern completion in hippocampus. J. Neurosci. 9:3915-3928; 1989.

21. Mizumori, S. J. Y.; Williams, J. D. Directionally-selective mnemonic properties of neurons in the lateral dorsal nucleus of the thalamus of rats. J. Neurosci. 13:4015-4028; 1993.

22. Muller, R. U.; Bostock, E.; Taube, J. S.; Kubie, J. L. On the directional properties of hippocampal place cells. J. Neurosci. 14:7235-7251; 1994.

23. Nudo, R. J.; Milliken, G. W.; Jenkins, W. M.; Merzenich, M. M. Use-dependent alterations of movement representations in primary motor cortex of adult squirrel monkeys. J. Neurosci. 16:785-807; 1996.

24. O'Keefe, J.; Dostrovsky, J. The hippocampus as a spatial map. Preliminary evidence from unit activity in the freely-moving rat. Brain Res. 34:171-175; 1971.

25. O'Keefe, J.; Nadel, L. The Hippocampus as a Cognitive Map. London: Oxford Univ.; 1978.

26. O'Keefe, J.; Speakman, A. J. Single unit activity in the rat hippocampus during a spatial memory task. Exp. Brain Res. 68:1-27; 1987.

27. Pyapali, G. K.; Turner, D. A. Increased dendritic extent in hippocampal CA1 neurons from Aged F344 rats. Neurobiol. Aging 17:601-611; 1996. 
28. Rapp, P.; Gallagher, M. Preserved neuron number in the hippocampus of aged rats with spatial learning deficits. Proc. Natl. Acad. Sci. USA 93:9926-9930; 1996.

29. Rapp, P.; Rosenberg, R.; Gallagher, M. An evaluation of spatial information processing in aged rats. Behav. Neurosci. 101:3-12; 1987.

30. Rasmussen, T.; Schliemann, T.; Sorensen, J. C.; Zimmer, J.; West, M. J. Memory impaired aged rats: No loss of principal hippocampal and subicular neurons. Neurobiol. Aging 17:143-147; 1996.

31. West, M. J.; Amaral, D. G.; Rapp, P. R. Preserved hippocampal cell number in aged monkeys with recognition memory deficits. Soc. Neurosci. Abstr. 19:599; 1993

32. Wilson, M. A.; McNaughton, B. L. Dynamics of the hippocampal ensemble code for space. Science 261:1055-1058; 1993.

33. Winocur, G. A neuropsychological analysis of memory loss with age. Neurobiol. Aging 9:487-494; 1988.

34. Witter M. P.; Griffioen, A. W.; Jorritsma-Byham, B.; Krijnen, J. L. M. Entorhinal projections to the hippocampal CA1 region in the rat: an underestimated pathway. Neurosci. Lett. 85:193-198; 1988. 